\title{
An enhanced adaptive non-local means algorithm for Rician noise reduction in magnetic resonance brain images
}

\author{
Kaixin Chen ${ }^{1}$, Xiao Lin ${ }^{1}$, Xing Hu', Jiayao Wang ${ }^{1}$, Han Zhong ${ }^{1}$ and Linhua Jiang ${ }^{1,2^{*}}$
}

\begin{abstract}
Background: The Rician noise formed in magnetic resonance (MR) imaging greatly reduced the accuracy and reliability of subsequent analysis, and most of the existing denoising methods are suitable for Gaussian noise rather than Rician noise. Aiming to solve this problem, we proposed fuzzy c-means and adaptive non-local means (FANLM), which combined the adaptive non-local means (NLM) with fuzzy c-means (FCM), as a novel method to reduce noise in the study.
\end{abstract}

Method: The algorithm chose the optimal size of search window automatically based on the noise variance which was estimated by the improved estimator of the median absolute deviation (MAD) for Rician noise. Meanwhile, it solved the problem that the traditional NLM algorithm had to use a fixed size of search window. Considering the distribution characteristics for each pixel, we designed three types of search window sizes as large, medium and small instead of using a fixed size. In addition, the combination with the FCM algorithm helped to achieve better denoising effect since the improved the FCM algorithm divided the membership degrees of images and introduced the morphological reconstruction to preserve the image details.

Results: The experimental results showed that the proposed algorithm (FANLM) can effectively remove the noise. Moreover, it had the highest peak signal-noise ratio (PSNR) and structural similarity (SSIM), compared with other three methods: non-local means (NLM), linear minimum mean square error (LMMSE) and undecimated wavelet transform (UWT). Using the FANLM method, the image details can be well preserved with the noise being mostly removed.

Conclusion: Compared with the traditional denoising methods, the experimental results showed that the proposed approach effectively suppressed the noise and the edge details were well retained. However, the FANLM method took an average of $13 \mathrm{~s}$ throughout the experiment, and its computational cost was not the shortest. Addressing these can be part of our future research.

Keywords: MR brain images, Rician noise reduction, Median absolute deviation (MAD), Adaptive non-local means (NLM)

\footnotetext{
* Correspondence: honorsir@yandex.com

'Shanghai Key Lab of Modern Optical Systems, School of Optical-Electrical

and Computer Engineering, University of Shanghai for Science and

Technology, 200093, P, Shanghai R, China

${ }^{2}$ School of Medicine, Stanford University, 269 Campus Drive, Stanford, CA

94305, USA
}

(c) The Author(s). 2020 Open Access This article is distributed under the terms of the Creative Commons Attribution 4.0 International License (http://creativecommons.org/licenses/by/4.0/), which permits unrestricted use, distribution, and reproduction in any medium, provided you give appropriate credit to the original author(s) and the source, provide a link to the Creative Commons license, and indicate if changes were made. The Creative Commons Public Domain Dedication waiver (http://creativecommons.org/publicdomain/zero/1.0/) applies to the data made available in this article, unless otherwise stated. 


\section{Background}

In recent years, magnetic resonance imaging, as a commonly used medical imaging technology, has played an important role in medical diagnosis and brings great convenience to medical workers. The accuracy of clinical diagnosis is dependent on the quality of the MR image, but the noise generated during the imaging process reduces the quality of the MR image. So the noise made the boundaries of some tissue structures blurred [1], which resulted in increased the difficulty in recognizing the image details. Therefore, MR images denoising is a crucial step.

Currently there are many different denoising methods, for example the wavelet transform [2-5], anisotropic diffusion filter (ADF) $[6,7]$, linear minimum mean square error (LMMSE) [8, 9] and the maximum likelihood approach [10, 11]. However, most of the denoising algorithms are only suitable for Gaussian noise, and ignored the influence of Rician noise in MR images. Recently, the non-local means algorithm (NLM) proposed by Buades et al. [12] utilized the redundancy of the image and takes its weighted average to reduce the noise. The NLM algorithm was designed to remove Gaussian noise, but when it was applied to remove the noise in MR images, some researchers found the NLM algorithm was better than the Gaussian filter, Wiener filter and other traditional filters. In order to speed-up the NLM filter, Coupe et al. [13] proposed block implementation and parallel computation to optimize the filter and reduce the calculation cost. Thaipanich et al. [14] used singular value decomposition to classify images into blocks, and adjusted the window size adaptively based on the block classification results. However, both methods were based on the assumption that the MR image noise was modeled as the Gaussian distribution, and were not suitable for Rician noise. A new method is needed for MR images with Rician noise.

In this paper, we proposed an adaptive NLM approach for the distribution of Rician noise: this approach is called FANLM. As we mentioned above, the proposed methods in $[13,14]$ were based on the assumption that the noise is modeled as the Gaussian distribution. To get accurate results, the proposed method used the improved MAD estimator for Rician noise to estimate the characteristics of each pixel in the MR images, and selected the optimal search window size according to the estimated noise variance. Moreover, in order to better preserve the details, the fuzzy c-means approach was used before denoising to divide the membership degree of MR image. Additionally, we used the improved adaptive NLM algorithm for denoising which designed three window size as small, medium, and large, and selected the optimal search window size for each pixel by the improved MAD estimator for Rician noise.

In order to verify the superiority of the proposed approach, we experimented with simulating human brain MR image data and real human brain $M R$ data, and compared the proposed approach with the traditional non-local means (NLM), linear minimum mean square error (LMMSE) and undecimated wavelet transform (UWT). The experimental results showed that the proposed method FANLM can better preserve the image details and remove noise, and the computational cost was also relatively reduced.

\section{Method}

\section{Brief review of non-local means}

The NLM filter exploits the data redundant among the patches in noisy images. In the filtered images, each pixel is a weighted average of non-local pixels. The mathematical expression of image noise can be expressed as $v(i)=u(i)+\eta(i)$, where $v(i)$ and $u(i)$ are the noisy and noise-free image pixels at pixel $i$, respectively, where $\eta(i)$ is a noise sample. By setting a search area, the estimated pixel value $\hat{u}_{N L M}(i)$ can be obtained by calculating the weighted average of the pixel values in the entire image, and is defined as follows:

$$
\hat{u}_{N L M}(i)=\sum_{j \in S_{i}} w(i, j) v(j)
$$

where the weight value $w(i, j)$ is determined by the similarity of the pixels $i$ and $j$ in the search domain $S_{i}$. The weights are defined as:

$$
w(i, j)=\frac{1}{z(i)} e^{-\frac{\left\|p\left(N_{i}\right)-p\left(N_{j}\right)\right\|_{2, a}^{2}}{h^{2}}}
$$

Where,

$$
z(i)=\sum_{j} e^{-\frac{d(i, j)}{h^{2}}}
$$

In Eq. (2), $p\left(N_{i}\right)$ and $p\left(N_{j}\right)$ are the patches centered on pixel $i$ and $j .\left\|p\left(N_{i}\right)-p\left(N_{j}\right)\right\|_{2, a}^{2}$ is the Euclidean distance between two patches weighted by a standard deviation $a$ of the Gaussian kernel. The smoothing parameter $h$ controls the smoothness of noise, and $z(i)$ is the normalized factor.

B. Kang et al. [15] and h. Bhujle et al. [16] both adapted the local edge structures by changing the weight function. The difference is that B. Kang et al. proposed 
the expression of attenuation coefficient as a function of edge degree [15], which resulted in a modified weight function. They did not use the regular Euclidean distance in the calculation of weight function and calculated the distance between blocks based on the edge graph. Therefore, the weight function had changed automatically along with the calculation of similar distances. If the filtering parameters of the NLM method, e.g. the search window, the sliding window size, and the pixel weight, are fixed in the experiment, the denoising result will not be ideal, and image edges also won not be well retained. Therefore, we proposed a method that adaptively selected the filtering parameters based on noise variations.

\section{Brief review of fuzzy c-means}

The fuzzy c-means (FCM) was proposed [17] since the traditional clustering method cannot divide image memberships well. The basic idea is to divide $\mathrm{N}$ vectors $x_{i}(i=$ $1,2,3 \ldots N)$ into $C$ fuzzy groups and then determine the clustering center of each class, so as to minimize the similarity between different classes. The FCM algorithm is based on the minimization of the following objective functions:

$$
J_{F C M}=\sum_{i=1}^{C} \sum_{j=1}^{N} \mu_{i j}^{m}\left\|x_{j}-k_{i}\right\|^{2}
$$

For the image $I=\left\{x_{i} \in I \mid i=1,2,3 \ldots N\right\}$, images with $\mathrm{N}$ pixels are divided into class $\mathrm{C}$ using the FCM algorithm. $\mu_{i j}$ is the value of membership of $i t h$ pixel in the cluster $j$. The clustering center $k_{i}$ and fuzzy membership $\mu_{i j}$ are expressed as:

$$
k_{i}=\frac{\sum_{j=1}^{N} \mu_{i j}^{m} x_{i}}{\sum_{j=1}^{N} \mu_{i j}^{m}}
$$

and

$$
\mu_{i j}=\frac{1}{\sum_{q=1}^{c}\left(\frac{\left\|x_{j}-k_{i}\right\|}{\left\|x_{j}-k_{q}\right\|}\right)^{2 /(m-1)}}
$$

satisfying the constraint conditions $\sum_{i=1}^{C} \mu_{i j}=1$.

However, FCM has its limitations: it can only segment simple texture images and is sensitive to noise; Thus, it cannot work well for noisy images. To solve this problem, s. Krinidis et al. [18] proposed a local fuzzy clustering algorithm (FLICM), but FLICM cannot make good use of the context information in the image for different local information. Gong, M et al. [19] and Maoguo, G et al. [20] made some improvements to FLICM, but the computational cost was still high.
Tao Lei et al. [21] put forward an improved robust FCM algorithm (FRFCM) which required less computational cost and was immune to noise. The FRFCM adopted a new approach that clustered the gray histogram of morphological reconstruction, and the membership degree of pixels were obtained through iteration. The FRFCM used the Lagrange multiplier to convert optimization problems into unconstrained optimization problems. The objective functions are as follows:

$$
J_{M}=\sum_{i=1}^{C} \sum_{j=1}^{N} \mu_{i j}^{m}\left\|x_{j}-v_{i}\right\|^{2}-\lambda\left(\sum_{j=1}^{N} \mu_{i j}-1\right)
$$

The main improvements of the FRFCM were twofold. First, morphological reconstruction was introduced into FCM to smooth the image, which improved the robustness of the algorithm and preserved the details of the image. Second, the FRFCM modified the classification of membership by using a faster filter instead of the slower distance computation between pixels in local spatial neighbors and clustering centers.

\section{Proposed method}

The traditional NLM denoising algorithm used a fixed size search window for each pixel. However, image pixels may be located in smooth or non-smooth areas. Suppose the pixel is in a non-smooth or textured area and the size of search window is still big, then denoising area will become more blurred due to the mean calculation; Similarly, if the smoothed area is filtered with a small search window, the variance of the filtered area will be much different from that of the original image. Therefore, we need to take into consideration the regional characteristics of each pixel and select the best size of search window based on the estimated variance of the search region. The proposed method selected the best window size adaptively: small(s), medium $(\mathrm{m})$, large(l). To accurately obtain the image features of each region, we need to estimate the noisy image to obtain the noise variance. Our proposed FANLM method is to estimate the noise variance by the improved MAD estimator for Rician noise. In order to better preserve the details, we used FRFCM to cluster brain MR images to determine pixels membership in the region of interest. Then, the FANLM denoised the brain MR image based on the estimated image variance and adaptively selected optimal search window size.

\section{Estimation of Rician noise}

The noise can be estimated by using methods based on wavelet transform [22] or principal component analysis (PCA) [23]. The method based on PCA is suitable for weak texture images but not so good for Rician noise 


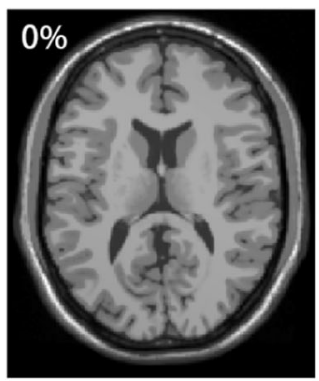

(a)

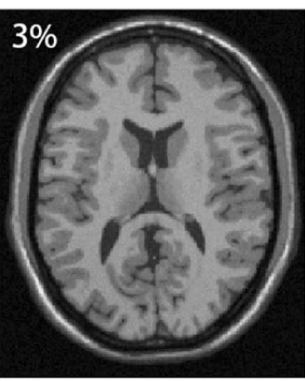

(b)

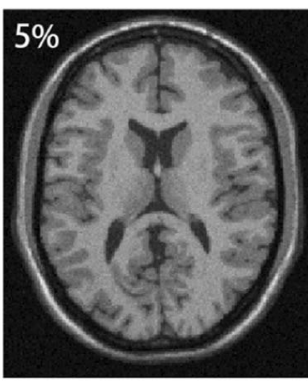

(c)

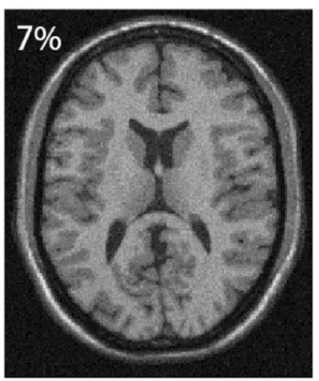

(d)

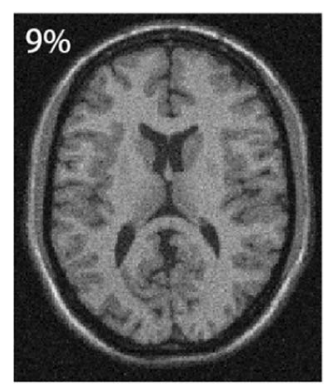

(e)

Fig. 1 Brain MR noisy images with 0, 3, 5, 7, 9\% noise leve

estimation. In the wavelet transform domain, we need to filter the image in the horizontal and vertical directions respectively to achieve wavelet multi-resolution decomposition. The divided sub-bands include LL, HL, LH and $\mathrm{HH}$ sub-bands, where the $\mathrm{HH}$ sub-band is composed of the wavelet noise coefficient which generated after convolution [24]. So the median absolute deviation estimator used the wavelet coefficients of the $\mathrm{HH}$ sub-band to estimate the image noise. The estimated standard deviation expression is as follows:

$$
\hat{\sigma}=\frac{\operatorname{median}(|H H|)}{0.6745}
$$

However, the noise estimation method is suitable for Gaussian noise. An improvement of estimating Rician noise is proposed by Guan, K et.al [25].. The main improvement depended on a fixed-point formula for the signal-to-noise ratio and the correction factor $\zeta$. And the variance of the amplitude image can be expressed as:

$$
\sigma_{n}^{2}=\zeta(\theta) \hat{\sigma}^{2}
$$

Where $\theta \equiv \mathrm{SNR}, \hat{\sigma}$ is estimated by the MAD for the initialization process and also provides a correction factor $\zeta$ :

$$
\zeta(\theta)=2+\theta^{2}-\frac{\pi}{8} \exp \left(-\frac{\theta^{2}}{2}\right)\left(\left(2+\theta^{2}\right) I_{0}\left(\frac{\theta^{2}}{4}\right)+\theta^{2} I_{1}\left(\frac{\theta^{2}}{4}\right)\right)^{2}
$$

$I_{1}$ is the first-order modified Bessel function that iterated through the correction factor until it converges, or reached a given number. The iteration would converge When $\left|\theta_{i}-\theta_{i-1}\right| \leq \varepsilon, \varepsilon=1.0 \times 10^{-8}$, and the iterative correction scheme can be expressed as:

$$
\theta_{i}=\sqrt{\zeta\left(\theta_{i-1}\right)\left(1+\frac{\bar{m}_{0}}{\hat{\sigma}^{2}}\right)-2}
$$

Where $\bar{m}_{0}$ is the average signal of the data, and $\hat{\sigma}$ is the average noise difference calculated by MAD estimator.

\section{Adaptive NLM to Rician noise}

The basic idea of this section is to obtain the optimal size of the search window according to the variance of original images and filtered images. With the traditional NLM algorithm, the noisy images are represented as $f_{i}^{s}, f_{i}^{m}$ and $f_{i}^{l}$ respectively, and the corresponding variance of each region is expressed as $\sigma_{f, s}^{2}, \sigma_{f, m}^{2}$ and $\sigma_{f, l}^{2}$. The estimated variance $\hat{\sigma}_{n}^{2}$ was obtained by using the improved MDA method for the Rician noise, and then the estimated variance of the original image can be expressed as:

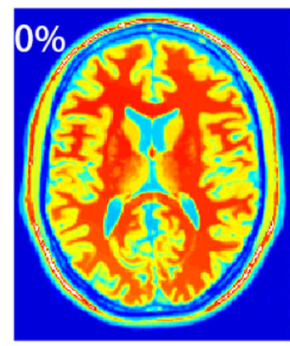

(a)

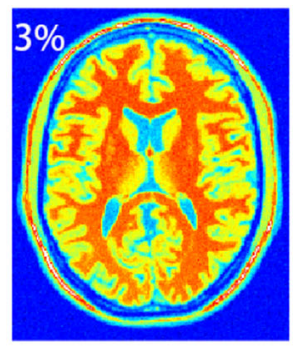

(b)

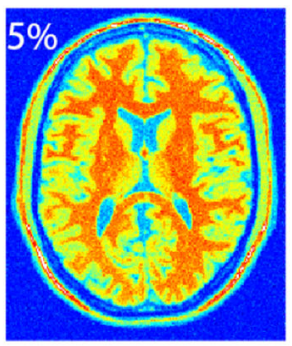

(c)

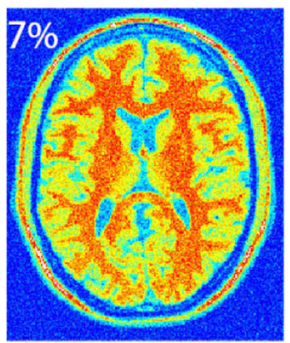

(d)

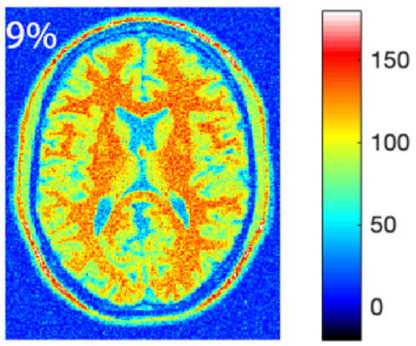

(e)

Fig. 2 Colored Brain MR noisy images with 0, 3, 5, 7, 9\% noise leve 


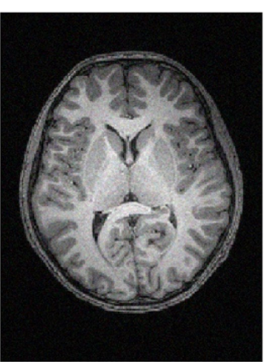

(a)

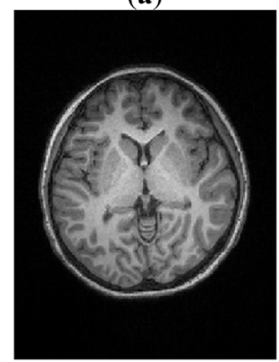

(e)

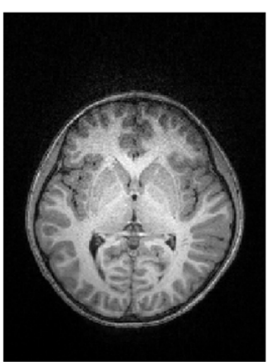

(b)

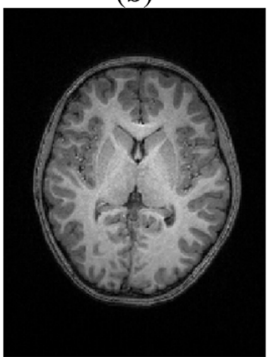

(f)

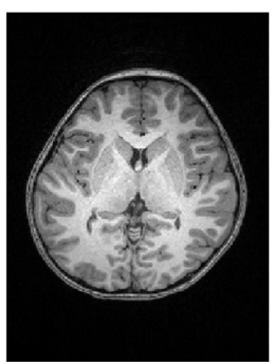

(c)

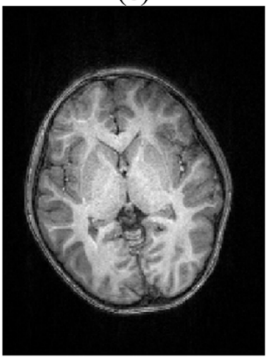

(g)

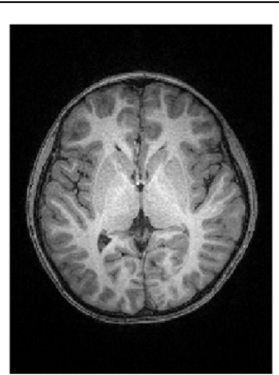

(d)

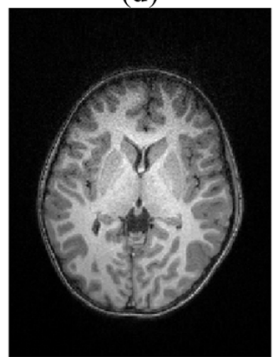

(h)

Fig. 3 Real human brain MR images

$$
\hat{\sigma}_{g, c}^{2}=\max \left(\sigma_{f, c}^{2}-\hat{\sigma}_{n}^{2}, 0\right)
$$

Where $c \in\{s, m, l\}$. We used the traditional NLM algorithm to filter the image. The filtered images obtained by small, medium and large search window are denoted as $\hat{u}_{N L M}^{s}, \hat{u}_{N L M}^{m}$ and $\hat{u}_{N L M}^{l}$. Then we can estimate the noise variance of the filtered image using the improved MAD estimator for Rician noise. The variances in the small, medium and large regions are expressed as $\hat{\sigma}_{N L M, s}^{2}, \hat{\sigma}_{N L M, m}^{2}$ and $\hat{\sigma}_{N L M, l}^{2}$. The optimal search window size is expressed by the following expression:

$$
\operatorname{size}\left(S_{i}^{b e s t}\right)=\operatorname{argmin}_{c}\left\{\left|\hat{\sigma}_{g, c}^{2}-\hat{\sigma}_{N L M, c}^{2}\right|\right\}
$$

In order to further improve the quality of the filtered image, we added FCM as a subsequent processing step. Traditional FCM is sensitive to noise and cannot remove noise very well. We used the improved and robust FCM algorithm (FRFCM) proposed by Tao Lei et al. [20]. The FRFCM algorithm used local membership filter instead of filtering the distance between the pixel and cluster center in traditional FCM algorithm, and it was able to speed-up the FCM algorithm. At the same time, the morphological reconstruction was used to smooth the image, and the processed image edge details were enhanced.

\section{Datasets}

\section{Dataset 1}

The experimental data in this part was downloaded from the BrainWeb [26]. We downloaded the T1-weighted brain MR images which are corrupted with $0,3,5,7$, and $9 \%$ of Rician noise, and the MR image size is $181 \times$ $217 \times 181$ voxels with $1 \mathrm{~mm}$ slice thickness. Different level of noisy MR images are shown in Fig. 1.

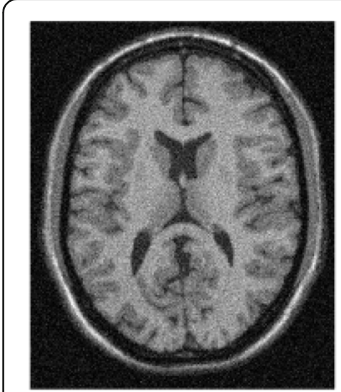

(a) $9 \%$

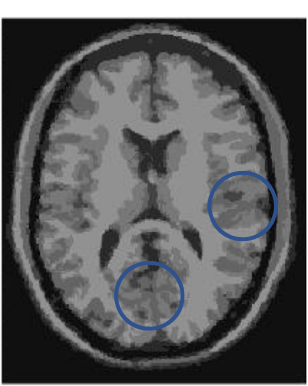

(b) UWT

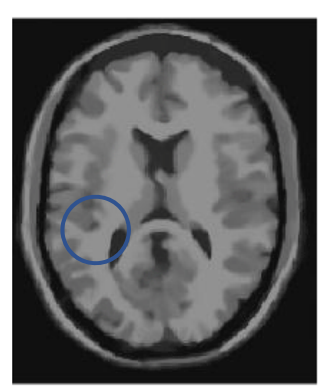

(c) LMMSE

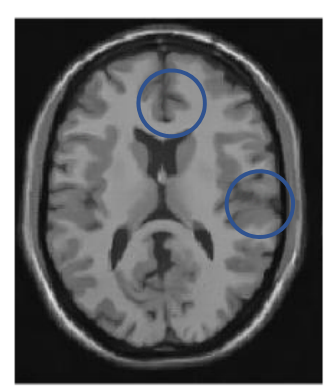

(d) NLM

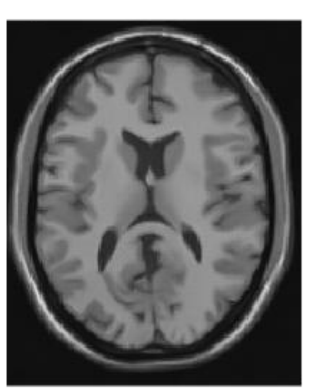

(e) FANLM

Fig. 4 Experimental results of different methods. (a) corrupted MR image with 9\% Rician noise; (b) UWT; (c) LMMSE; (d) NLM; (e) FANLM 


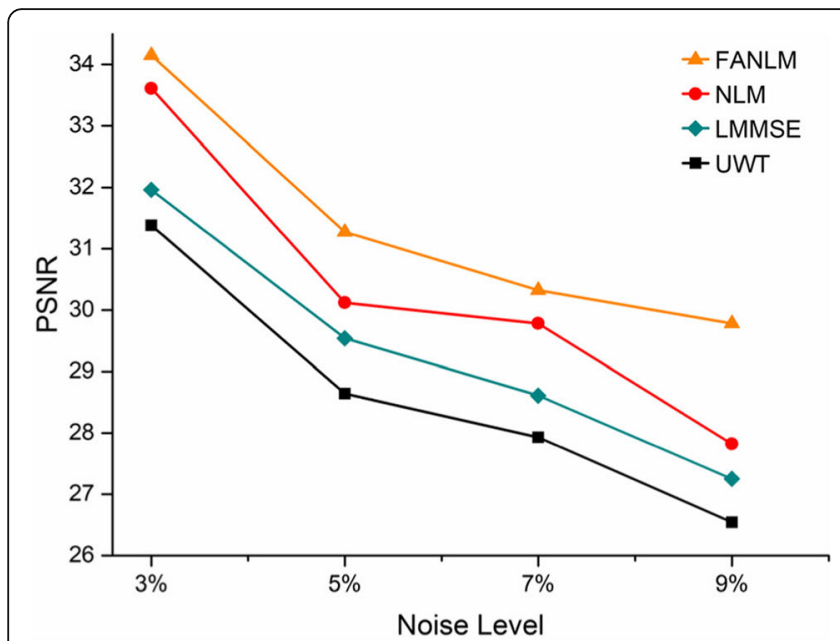

(a)

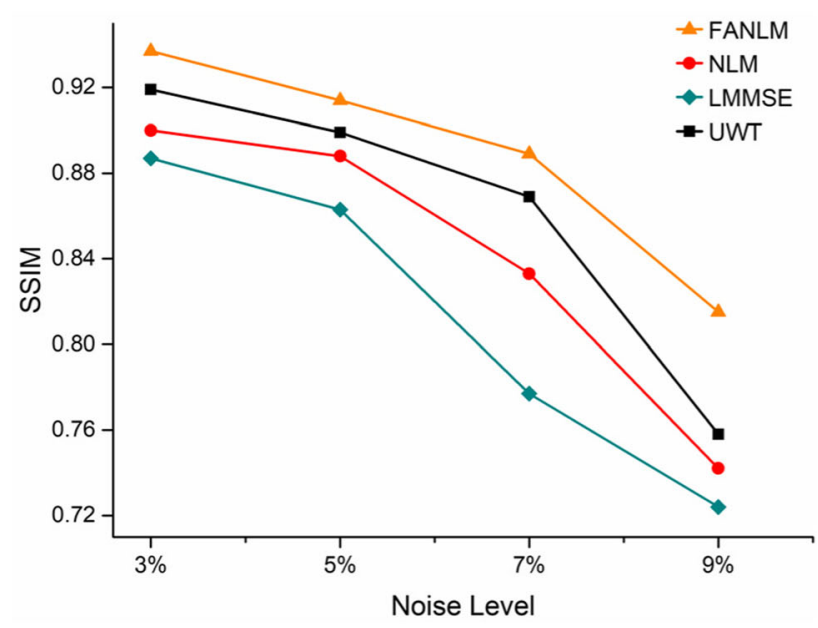

(b)

Fig. 5 Comparison of the PSNR and SSIM of four methods. (a) PSNR; (b) SSIM

Since the naked eye cannot distinguish the difference of noise levels very well, we used colormap to dye the images. As shown in Fig. 2, we can clearly see that as the noise level increases, the boundaries of the MR images became more and more blurred, and some structural details have been lost. So it is necessary to remove the noise in MR images.

\section{Dataset 2}

The dataset 2 is real human brain MR images collected by using the Siemens MAGNETOM Prisma $3 \mathrm{~T}$ magnetic resonance imaging system in the State Key Laboratory of Cognitive Neuroscience and Learning at Beijing Normal University. The data consisted of 8 T1-weighted brain MR images as shown in Fig. 3, and the size of images is $192 \times 256 \times 170$ with resolution of $1 \mathrm{~mm}$.

\section{Selecting size of search window}

Selection of the search window size through experiments have been discussed in some articles. The experiment in Buades's study [27] showed that we can use $7 \times 7$ or $9 \times 9$ (window size) when processing gray images. And for color images with less noise, we can use $3 \times 3$ or $5 \times 5$ window size. Some researchers $[28,29]$ suggest that square search window in the range of $9 \times 9$ to $21 \times 21$ can be used for optimal algorithm and better experimental results. We found that it was difficult to remove noise by using a smaller window in our experiments. Therefore, we designed different sizes of the search window when processing different noisy images. In our proposed NLM approach, we chose $7 \times 7$ for small window, $13 \times 13$ for medium window, and $21 \times 21$ for large window.

\section{Results}

In our study, four methods were used to denoise the noisy MR images: the proposed fuzzy c-means and adaptive non-local means (FANLM), non-local means (NLM), linear minimum mean square error (LMMSE), undecimated wavelet transform (UWT).

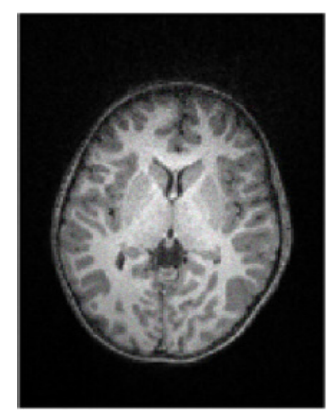

(a) $9 \%$

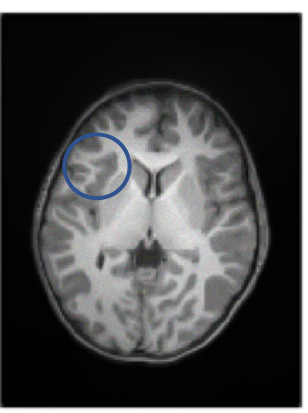

(b) UWT

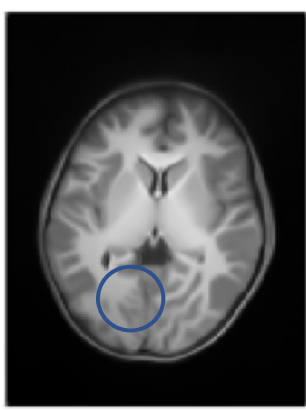

(c) LMMSE

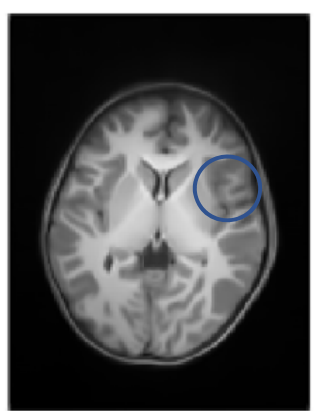

(d) NLM

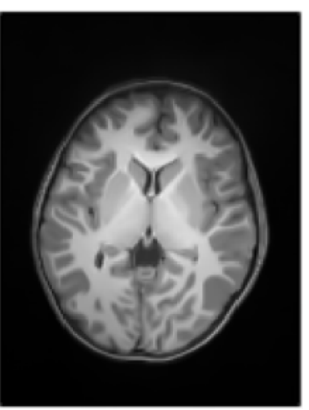

(e) FANLM

Fig. 6 Experimental results of different methods. (a) corrupted MR image with 9\% Rician noise; (b) UWT; (c) LMMSE; (d) NLM; (e) FANLM 


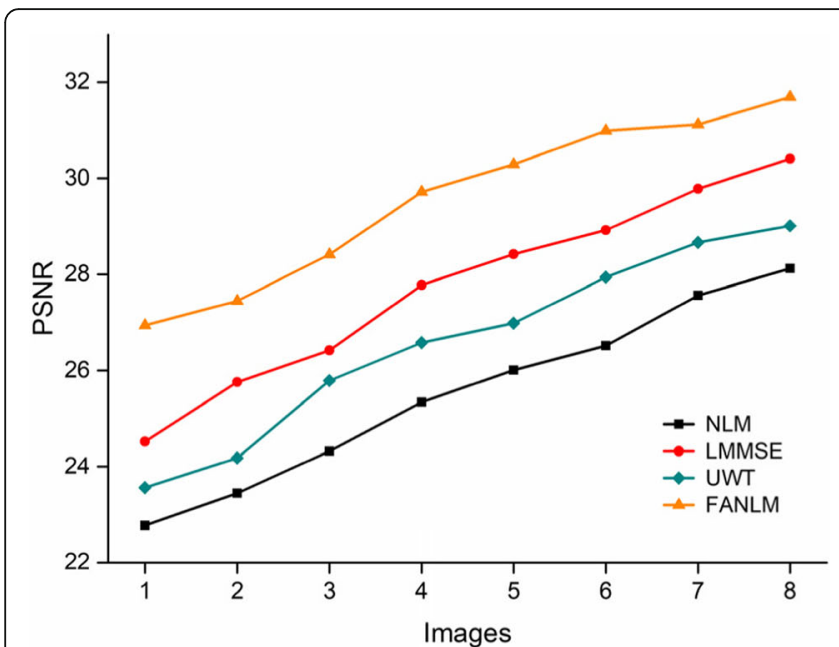

(a)

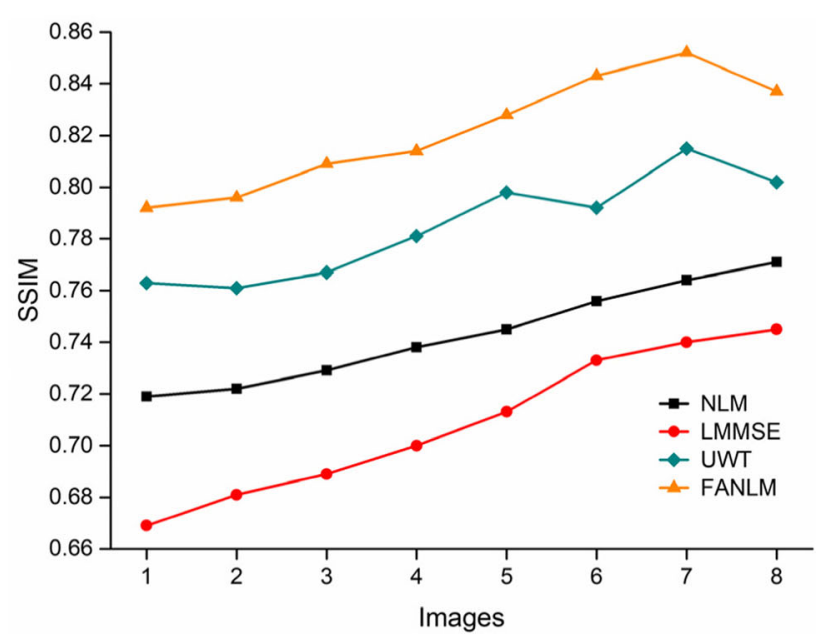

(b)

Fig. 7 Comparison of the PSNR and SSIM of four methods. (a) PSNR; (b) SSIM

All the experiments were conducted in MATLAB on Intel(R) Core i5-3470, 3.40Ghz CPU with 4GB RAM.

To evaluate and analyze the quality of the denoised MR images, two criteria were used: the structural similarity (SSIM) and the peak signal-to-noise ratio (PSNR). The experiment results were divided into two parts, one is the analysis result of the MR images downloaded from BrainWeb with noise levels of 3, 5, 7 and 9\% respectively, and the other is the real data images acquired by MAGNETOM Prisma.

\section{Dataset 1}

We denoised the MR images with noise levels of 3, 5, 7 and $9 \%$ by using the proposed FANLM method and the NLM, LMMSE and UWT respectively. As shown in Fig. 4, it displays the original MR image (noise level of 9\%) and the denoise results obtained by the four methods.

As shown in Fig. 4, compared with the FANLM, the UWT removed most of the noise, but the details were also removed in the meantime, and the structural details were not well preserved. The problems of LMMSE are that the edge details were not preserved as well, and ringing artifacts occurred in some parts

Table 1 Comparison of computational time on datasets 1

\begin{tabular}{lllll}
\hline $\begin{array}{l}\text { Denoising } \\
\text { methods }\end{array}$ & \multicolumn{4}{l}{ Computational time $(\mathrm{s})$} \\
\cline { 2 - 5 } & $3 \%$ & $5 \%$ & $7 \%$ & $9 \%$ \\
\hline FANLM & 5.85 & 6.21 & 8.14 & 9.43 \\
NLM & 9.52 & 11.92 & 12.67 & 14.39 \\
LMMSE & 2.12 & 2.88 & 3.48 & 4.92 \\
UWT & 2.78 & 3.56 & 4.32 & 5.14 \\
\hline
\end{tabular}

of MR images. NLM used the fixed parameters and the same size of search window to denoise different regions, which resulted in excessive smoothing in some texture regions. However, the proposed method FANLM method was able to preserve structural details effectively. In order to see the difference in denoising more clearly, we circled these different places in the image.

The above analysis of denoising results are only compared to the perspective of vision. In order to further verify the effectiveness of the proposed algorithm, the Fig. 5 shows the PSNR and SSIM obtained by the four methods when the original image had 3, 5, 7, and 9\% R Rician noise levels. The results showed that our proposed method FANLM achieved the optimal PSNR and SSIM at different noise levels.

\section{Dataset2}

In this part, the real brain MR images were filtered by the NLM, LMMSE and UWT and FANLM method. Figure 6 presents the results in terms of visual quality, and Fig. 7 lists the PSNR and SSIM values.

We cannot intuitively tell the denoising performance difference based on the denoised image. But if we carefully observe and compare the edge details of the denoised image, we find that FANLM can better retain the edges and while suppressing noise well.

Figure 7 shows the PSNR and SSIM values of eight real brain MR images when using the four different methods. The results show that our proposed method achieved the highest PSNR and SSIM, which 
Table 2 Comparison of computational time on dataset 2

\begin{tabular}{lllllllll}
\hline $\begin{array}{l}\text { Denoising } \\
\text { method }\end{array}$ & \multicolumn{1}{l}{ Computational time $(\mathrm{s})$} & & & & \\
\cline { 2 - 9 } & Image 1 & Image 2 & Image 3 & Image 4 & Image 5 & Image 6 & Image 7 & Image 8 \\
\hline FANLM & 16.93 & 17.35 & 14.43 & 16.30 & 17.73 & 18.85 & 17.19 & 16.93 \\
NLM & 30.52 & 35.26 & 29.51 & 31.48 & 32.59 & 36.71 & 34.85 & 32.67 \\
LMMSE & 10.76 & 12.47 & 10.79 & 11.25 & 12.74 & 14.53 & 13.40 & 12.38 \\
UWT & 13.27 & 14.89 & 12.12 & 13.76 & 15.10 & 18.76 & 15.32 & 14.17 \\
\hline
\end{tabular}

means that our method is superior to the other approaches.

\section{Discussion}

Quantitative results are compared in the above section, and the results show that the proposed method can not only remove the noise effectively but also well preserve the detailed structures. However, the computational cost of the proposed method FANLM is only superior to that of NLM. In the future, we will make efforts to decrease computational complexity.

In Table 1, we list the computational times on datasets 1 for the four methods and with 3, 5, 7, and 9\% Rician noise levels. In terms of PSNR and SSIM, the proposed FANLM in this paper is better than other methods. However, in terms of running time, it is obvious that the computational cost of this method is still not superior due to its complexity of calculating the weight and adaptively choosing the optimal window size. Although the computation time of FANLM is shorter than that of NLM, it is still longer than those of LMMSE and UWT. In the future, we will make efforts to decrease the computational complexity.

We also compared the computational cost on dataset 2 , and the same problem existed. In Table 2, we list the computational costs of eight MR images. As stated above, the FANLM takes longer to calculate and has a computational burden due to its complexity of selecting the window size. Therefore, we need to improve the computation time in future works.

Overall, the following work has been completed in this paper. A novel method for denoising Rician noise in MR images was proposed. The adaptive NLM method can independently select the optimal search window size for each pixel according to the noise variance that is estimated by the improved MAD estimator. The FRFCM can divide the membership degrees of MR images to preserve the details well. However, the FANLM method takes a long time to select the window size, and the steps are slightly cumbersome. All these issues can be subjects in future studies.

\section{Conclusions}

As described in this paper, we proposed an improved method to suppress the Rician noise of MR images. We designed three window sizes, and the optimal size of search window is selected according to the estimated region characteristics. By combining the FRFCM method with the adaptive NLM algorithm, we proposed the FANLM method to denoise the MR images. Using the FANLM method, the image details can be well preserved with the noise mostly being removed. The experimental results show that the proposed algorithm FANLM can effectively remove the noise and is better than the NLM, LMMSE and UWT methods.

\section{Abbreviations}

ADF: Anisotropic diffusion filter; FANLM: Fuzzy c-means and adaptive nonlocal means; FCM: Fuzzy c-means; FLICM: Local fuzzy clustering algorithm; FRFCM: Robust FCM algorithm; LMMSE: Linear minimum mean square error; MAD: Median absolute deviation; MRI: Magnetic resonance image; MSE: Mean square error; NLM: Non-local means; PCA: Principal component analysis; PSNR: Peak signal-to-noise ratio; SSIM: Structural similarity; UWT: Undecimated wavelet transform

\section{Acknowledgments}

We thank to professor Shaozheng Qin from State Key Laboratory of Cognitive Neuroscience and Learning, Beijing Normal University for his kindly help and partially technical assistant.

\section{Informed consent}

Written informed consent was obtained from all individual participants included in the study.

\section{Authors' contributions}

Conceptualization K.C., H.Z. and L.J.; methodology K.C.; software, formal analysis, writing and editing, K.C. and L.J.; supervision L.J.; resources, project administration, funding acquisition X.L. and L.J.; help revise manuscript X.L.; editing English grammar X.H. and J.W. All authors read and approved the final manuscript.

\section{Funding}

The research was supported by founds form the National Natural Science Foundation of China (No. 61775139 and No. 61872242). In the role of design of the study, collection data and writing manuscript.

The research was partially supported by National Key R\&D Program of China under Grant 2019YFB1705702. In the role of revising manuscript.

\section{Availability of data and materials}

The datasets 1 used in this study are publicly available from the BrainWeb [26]. The datasets 2 are available from the corresponding author on reasonable request.

Ethics approval and consent to participate

The procedures of consent and experiment were approved by local ethics that were in accordance with the standards of the Declaration of Helsinki, and written informed consents were obtained from study participants.

Consent for publication

The consent for publication have been obtained from participants. 


\section{Competing interests}

The authors declare no conflict of interest.

Received: 4 September 2019 Accepted: 27 December 2019

Published online: 06 January 2020

\section{References}

1. McJury M, Shellock F. Auditory Noise Associated With MR Procedures: A Review, vol. 2000:12. https://doi.org/10.1002/1522-2586(200007)12:1<37::AIDJMRI5>3.0.CO;2-1.

2. Luisier F, Blu T, Wolfe PJ. A CURE for Noisy magnetic resonance images: ChiSquare unbiased risk estimation. IEEE Trans Image Process. 2012;21(8):345466. https://doi.org/10.1109/TIP.2012.2191565.

3. Yang $X$, Fei B. A wavelet multiscale denoising algorithm for magnetic resonance (MR) images. Measurementence Technol. 2011;22(22):25803.

4. Ville DVD, Seghier ML, Lazeyras F, Blu T, Unser M. WSPM: wavelet-based statistical parametric mapping. Neuroimage. 2007;37(4):1205-17.

5. Aleksandra $P$, Wilfried $P$, Ignace $L$, Marc A. A versatile wavelet domain noise filtration technique for medical imaging. IEEE Trans Med Imaging. 2003; 22(3):323-31.

6. Karl K, Santiago AF. Noise-driven anisotropic diffusion filtering of MRI. IEEE Trans Image Process Publication IEEE Signal Process Soc. 2009:18(10):2265.

7. Rajan J, Jeurissen B, Sijbers J, Kannan K Denoising Magnetic Resonance Images Using Fourth Order Complex Diffusion. In: 2009 13th Int Mach Vis Image Process Conf, 2-4 Sept. 2009. pp 123-127. doi:https://doi.org/10. 1109/IMVIP.2009.29

8. Santiago AF, Marc N, Marek K, Shenton ME, Carl-Fredrik W. Restoration of DWI data using a Rician LMMSE estimator. IEEE Trans Med Imaging. 2008; 27(10):1389-403.

9. Golshan HM, Hasanzadeh RPR, Yousefzadeh SC. An MRI denoising method using image data redundancy and local SNR estimation. Magn Reson Imaging. 2013;31(7):1206-17.

10. Jeny R, Ben J, Marleen V, Johan VA, Jan S. Maximum likelihood estimationbased denoising of magnetic resonance images using restricted local neighborhoods. Phys Med Biol. 2011;56(16):5221.

11. Rajan J, Veraart J, Audekerke JV, Verhoye M, Sijbers J. Nonlocal maximum likelihood estimation method for denoising multiple-coil magnetic resonance images. Magn Reson Imaging. 2012;30(10):1512-8.

12. Buades A, Coll B, Morel J A non-local algorithm for image denoising. In: 2005 IEEE Comput Soc Conf Comput Vis Pattern Recognit (CVPR'05), 20-25 June 2005. pp 60-65 vol. 62. doi:https://doi.org/10.1109/CVPR.2005.38

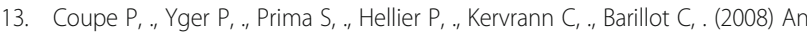
optimized blockwise nonlocal means denoising filter for 3-D magnetic resonance images. IEEE Trans Med Imaging 27 (4):425-441.

14. Thaipanich T. Kuo CCJ an adaptive nonlocal means scheme for medical image Denoising. In: Med Imaging: Image Process. 2010.

15. Kang B, Choi O, Kim JD, Hwang D. Noise reduction in magnetic resonance images using adaptive non-local means filtering. Electron Lett. 2013;49(5): 324-5.

16. Bhujle HV, Chaudhuri S. Laplacian based non-local means denoising of MR images with Rician noise. Magn Reson Imaging. 2013;31(9):1599-610.

17. Bezdek JC, Ehrlich R, Full W. FCM: the fuzzy c -means clustering algorithm. Comput Geosci. 1984;10(2):191-203.

18. Stelios K, Vassilios C. A robust fuzzy local information C-means clustering algorithm. IEEE Trans Image Process Publication IEEE Signal Process Soc. 2010;19(5):1328

19. Gong M, Zhou Z, Ma J. Change detection in synthetic aperture radar images based on image fusion and fuzzy clustering. Image Process IEEE Trans. 2012;21(4):2141-51.

20. Maoguo G, Yan L, Jiao S, Wenping M, Jingjing M. Fuzzy C-means clustering with local information and kernel metric for image segmentation. IEEE Trans Image Process. 2013;22(2):573-84.

21. Tao L, Jia X, Zhang $Y$, He L, Meng H, Nandi AK. Significantly fast and robust fuzzy $\mathrm{C}$-means clustering algorithm based on morphological reconstruction and membership filtering. IEEE Transactions on Fuzzy Systems PP. 2018;99:1-1.

22. Chang SG, Yu B, ., Vetterli M, . (2000) Spatially adaptive wavelet thresholding with context modeling for image denoising. IEEE Trans Image Process 9 (9): 1522-1531.

23. Liu X, Tanaka M, Okutomi M Noise level estimation using weak textured patches of a single noisy image. In: 2012 19th IEEE Int Conf Image Process,
30 Sept.-3 Oct. 2012. pp 665-668. doi:https://doi.org/10.1109/ICIP.2012. 6466947

24. Donoho $\mathrm{DL}$, Johnstone IM. Ideal spatial adaptation by wavelet shrinkage. Biometrika. 1994;81(3):425-55.

25. Guan KC, Basser PJ. Analytically exact correction scheme for signal extraction from noisy magnitude MR signals. J Magn Reson. 2006;179(2): 317-22.

26. BrainWeb: Simulated Brain Database. https://brainweb.bic.mni.mcgill.ca/. Access 11 Apr 2019

27. Buades A, Coll B, Morel JM. Image Denoising methods. A new nonlocal principle. SIAM Rev. 2010;52(1):113-47.

28. Ville DVD, Kocher M. SURE-based non-local means. IEEE Signal Process Lett. 2009:16(11):973-6.

29. Manjón JV, Carbonell-Caballero J, Lull JJ, García-Martí G, Martí-Bonmatí L, Robles M. MRI denoising using non-local means. Med Image Anal. 2008; 12(4):514-23.

\section{Publisher's Note}

Springer Nature remains neutral with regard to jurisdictional claims in published maps and institutional affiliations.
Ready to submit your research? Choose BMC and benefit from:

- fast, convenient online submission

- thorough peer review by experienced researchers in your field

- rapid publication on acceptance

- support for research data, including large and complex data types

- gold Open Access which fosters wider collaboration and increased citations

- maximum visibility for your research: over $100 \mathrm{M}$ website views per year

At BMC, research is always in progress.

Learn more biomedcentral.com/submission 